\title{
Pathogenicity and tissue distribution of grass carp reovirus after intraperitoneal administration
}

\author{
Hong-Ru Liang ${ }^{1}$, Yong-Gang Li ${ }^{1,2}$, Wei-Wei Zeng ${ }^{1}$, Ying-Ying Wang ${ }^{1}$, Qing Wang ${ }^{1 *}$ and Shu-Qin Wu ${ }^{1 *}$
}

\begin{abstract}
Grass carp reovirus (GCRV) is the causative agent of grass carp hemorrhage and causes significant loss of fingerlings. However, little is known about how the virus is distributed in organs and tissues. The aim of the present study was to investigate the distribution of different GCRV stains in tissues and organs of grass carp. The pathogenicity and tissue distribution of GCRV were monitored after intraperitoneal administration. The study showed a distribution of GCRV in different tissues and organs, particularly in the liver, spleen, kidney, intestine, and muscle, which had a higher number of viral RNA copies during the sixth to ninth days. The kidney had the highest numbers of viral RNA copies, as high as 24000 copies. Until the fourteenth day, nearly no viral RNA copies could be detected. This study defined the virus distribution in different tissues of grass carp inoculated by i.p. and supplied clues for the pathogenesis of GCRV.
\end{abstract}

Keywords: Distribution, GCRV, qRT-PCR

\section{Introduction}

Grass carp reovirus (GCRV), a fatal pathogen to aquatic animals, can provoke severe hemorrhagic disease in grass carp and causes a mortality rate of up to $85 \%$ during the summer months, when water temperatures range between $24^{\circ} \mathrm{C}$ and $30^{\circ} \mathrm{C}[1,2]$. GCRV infects grass carp, Ctenopharyngodon idellus, was found to be capable of infecting black carp Mylopharyngodon piceus, topmouth gudeon, Pseudorasbora parva, and rare minnow Gobiocypris rarus, and historically has resulted in large losses in freshwater fish culture [3]. The disease is one of the major diseases that damages the grass carp breeding industry and, every year, leads to at least 1 billion economic losses in China.

GCRV belong to genus Aquareovirus in the family Reoviridae. The GCRV is a noneveloped, icosahedral particle comprised of 11 double-stranded RNA genome segments surrounded by multiple concentric protein capsids [4]. There is little know about the genus Aquareovirus, but always focused on the molecular and structural biology of their structural proteins [5].

However, to date, there is no effective antiviral treatment against GCRV infection [6], and preventing these

\footnotetext{
* Correspondence: sunny_929@163.com; wushuqin001@21cn.com 'Pearl River Fisheries Research Institute, Chinese Academy of Fishery Sciences, Key Laboratory of Fishery Drug Development,Ministry of Agriculture, Key Laboratory of Aquatic Animal Immune Technology, Guangzhou 510380, China

Full list of author information is available at the end of the article
}

infectious diseases is still a great challenge in grass carp farming [7]. GCRV has been recognized as the most pathogenic amongst in the isolated aquareoviruses [8]. However, information on the tissue distribution and dynamic changes of GCRV in tissues and organs after infection by GCRV is lacking. Moreover, GCRV serves as a good model for studying viral replication and pathogenesis of Aquareovirus due to its high virulence [9].

In this study, grass carp were intraperitoneally inoculated (i.p.) with different GCRV strains, and the tissue distribution was analyzed. The GCRV-HZ08 stain was isolated form China's Zhejiang province and GCRV-CL stain was isolated form China's Hunan province, which have different virulence. A better understanding of the viral dynamics and distribution of the different GCRV strains in tissues will be helpful to control the disease.

\section{Methods}

Cells, virus and reagents

GSB cells were used for GCRV replication and grown in Eagle's minimum essential medium (MEM; Invitrogen, USA) supplemented with $10 \%$ fetal calf serum (FBS; HyClone, USA) at $37^{\circ} \mathrm{C}$.

The GCRV-HZ08 and GCRV-CL strains were obtained from the Pearl River Fishery Research Institute (Guangzhou, China). Grass carp, Sixteen- to eighteen-week-old, with a body length of $10 \pm 1.0 \mathrm{~cm}$ and an average weight of 


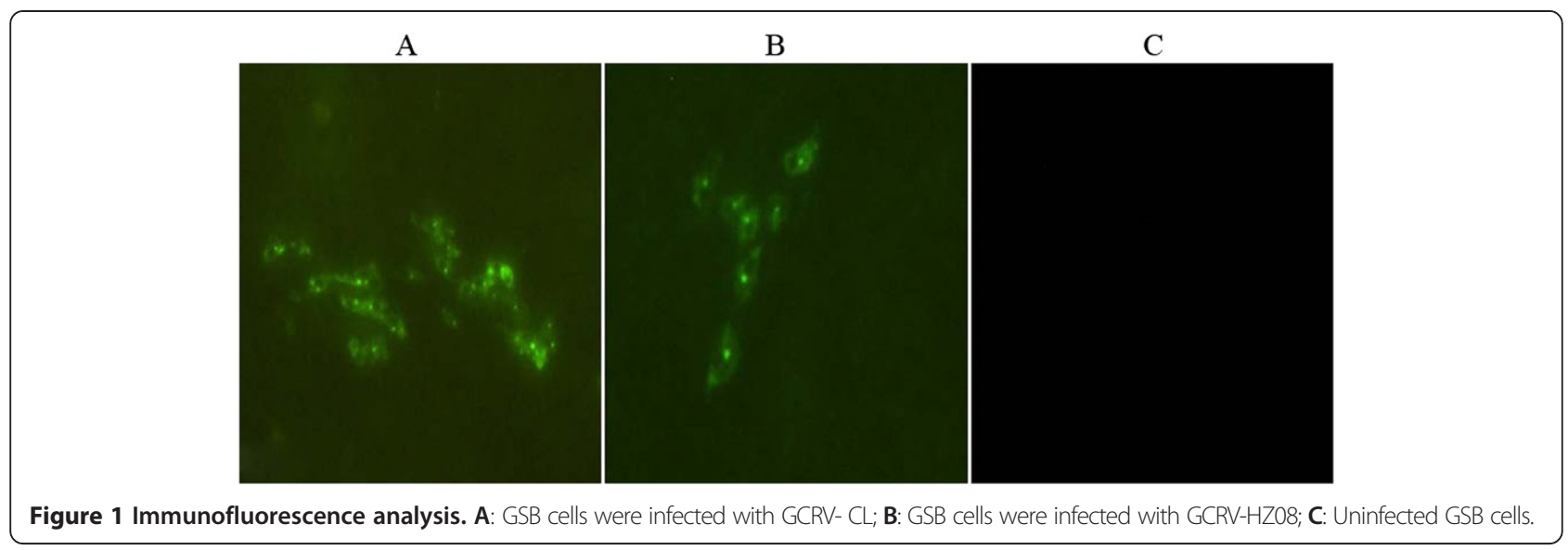

$20.0 \pm 1.1 \mathrm{~g}$, were obtained from a GCRV-free zone in NanHai (Guangdong, China).

\section{Immunofluorescence analysis}

The cells were infected with GCRV-HZ08 or GCRV-CL at a multiplicity of infection (MOI) of 0.5 . At $48 \mathrm{~h}$ postinfection, the infected cells were fixed with $4 \%$ paraformaldehyde for $20 \mathrm{~min}$ at room temperature and then treated with $0.1 \%$ Triton-X100. After washing 3 times with PBS, the cells were incubated with rabbit anti-grass carp reovirus antibody (1:1000), followed by FITCconjugated goat anti- rabbit IgG (Boster, China) (1:100). The infected cells were determined using a fluorescence microscope.

\section{Fish pathogenicity experiments}

Grass carps (ten in each group) were intraperitoneally (i.p.) injected with $0.2 \mathrm{~mL}$ of the appropriate GCRVHZ08 or GCRV-CL, and the control group was intraperitoneally (IP) injected with $0.2 \mathrm{~mL}$ of PBS. The grass

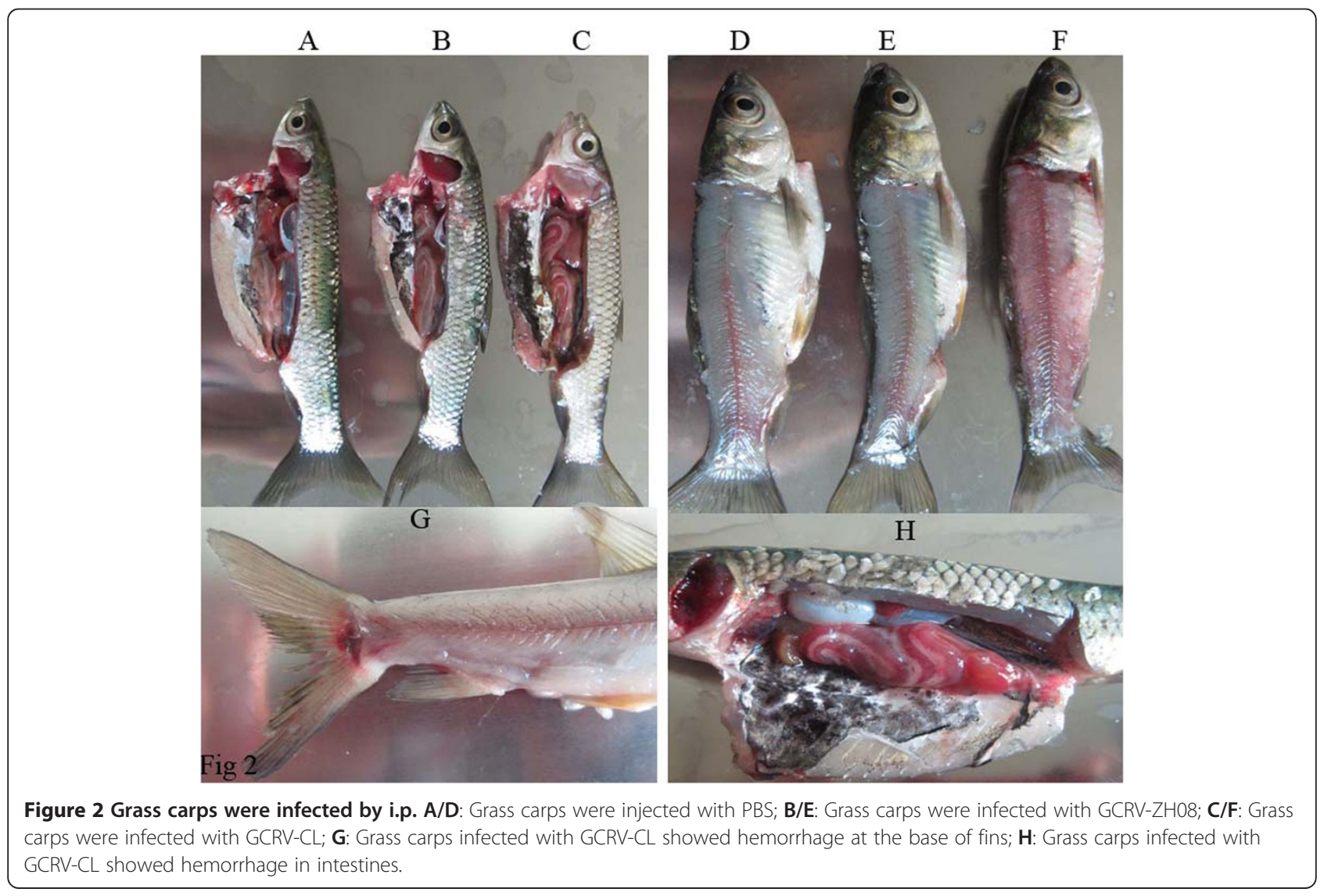




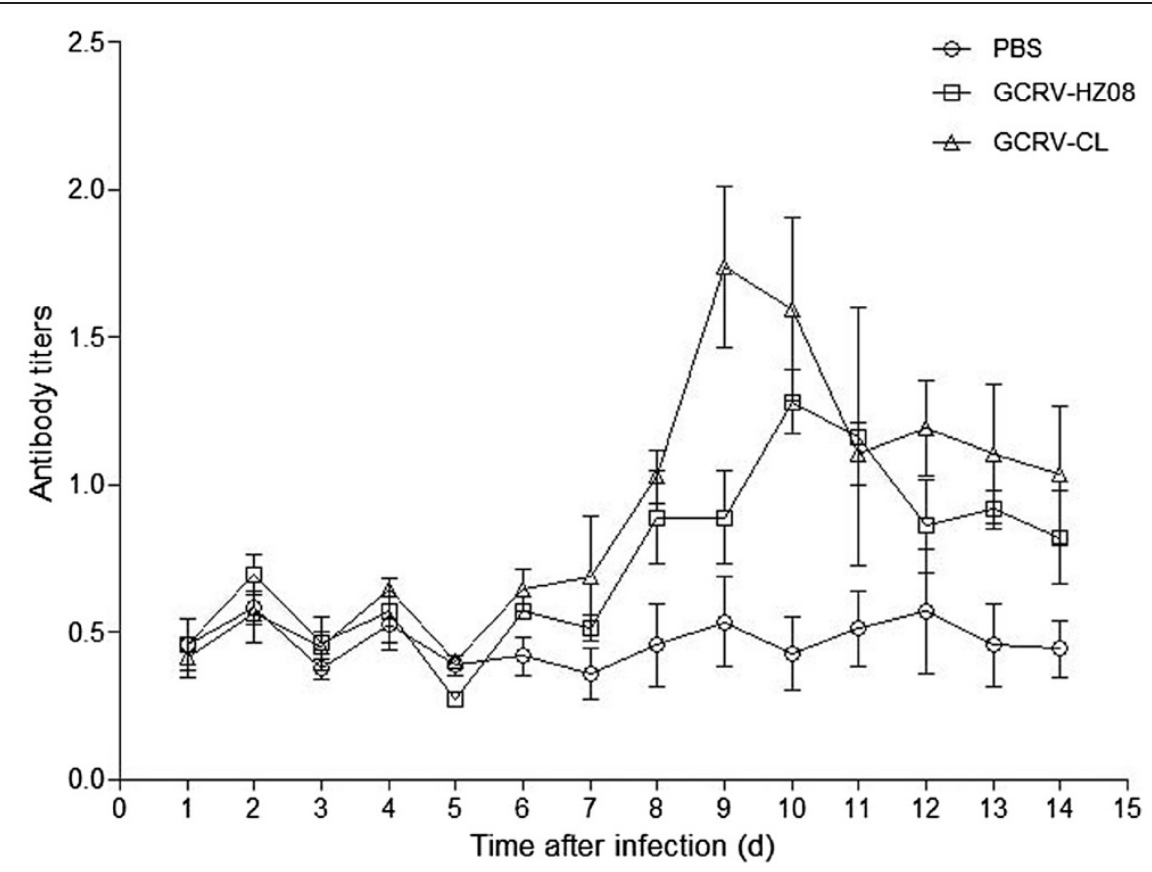

Figure 3 Titers of the serum were determined by using ELISA.

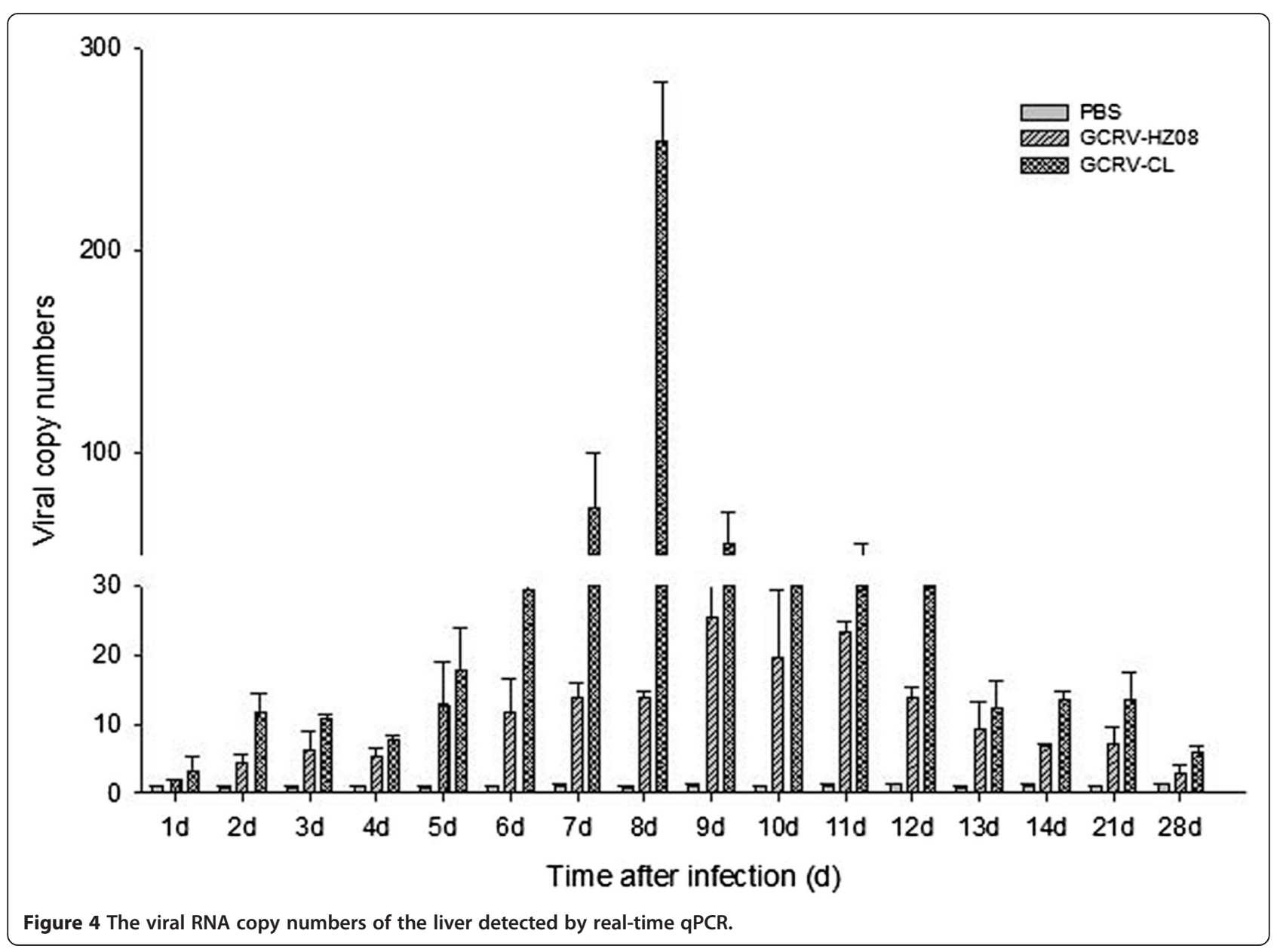


carps were monitored over three weeks for clinical signs of hemorrhagic disease.

\section{Tissue distribution of grass carp reovirus}

The grass carps were divided into three groups, with 60 fishes in each group: (i) grass carps intraperitoneally inoculated with $100 \mu \mathrm{l} \mathrm{GCRV-HZ08;} \mathrm{(ii)} \mathrm{grass} \mathrm{carps} \mathrm{intra-}$ peritoneally inoculated with $100 \mu \mathrm{l} \mathrm{GCRV-CL;} \mathrm{and} \mathrm{(iii)}$ grass carps intraperitoneally inoculated with sterile PBS as a negative control group.

\section{Detection of antibody}

The blood of three grass carps from each group were sampled, and the titers of the serum were determined using Enzyme-linked immunosorbent assay (ELISA). For ELISA, 96-well plates were coated with $70 \mathrm{ng}$ of the purified GCRV S10 protein per well at $4^{\circ} \mathrm{C}$ overnight. After all of the coated wells were blocked with $1 \%$ BSA, serum were loaded into the plate wells and incubated at $37^{\circ} \mathrm{C}$ for $2 \mathrm{~h}$. Anti-grass carp monoclonal antibody with HRP conjugate (1:10000 dilution in 1\% PBST) was then added and incubated at $37^{\circ} \mathrm{C}$ for $1 \mathrm{~h}$. Finally, 3,3,5,5'-tetramethylbenzidine (CoWin Biotech,
Beijing) was used for the color reaction, which was stopped with $\mathrm{H}_{2} \mathrm{SO}_{4}$. Each serum was assayed in duplicate with three repeats. The absorbance was determined at $450 \mathrm{~nm}$ using a spectrophotometer (Infinite M200 PRO, Tecan).

\section{Detection of viral RNA copy numbers by real-time qRT-PCR}

Three grass carps from each group were sampled from the first to fourteenth day, and then on the twenty-first and twenty-eighth day, and the different tissues were collected. All the samples were stored at $-80^{\circ} \mathrm{C}$ until use.

The viral RNA copy numbers were measured by quantitative real-time RT-PCR (qRT-PCR) as in a previous study. Briefly, total RNA was extracted using the Trizol reagent (Invitrogen, NY, USA) according to the manufacturer's protocol. The isolated RNA was then reverse transcribed using the First Strand cDNA Synthesis Kit (Takara, Japan). The cDNA was used as template for qRT-PCR performed using Premix Ex TaqTM (Perfect Real Time) (Takara, Japan) to assay samples with the following GCRV S7 gene-specific primers: F, 5'-ccaggaatcaatagcaatc-3', and R, 5'-cctgatataatcgctcttc-3'. The internal probe was 5'-cgataaccaccactacggctg-3'. The probe was labeled on its $5^{\prime}$ end

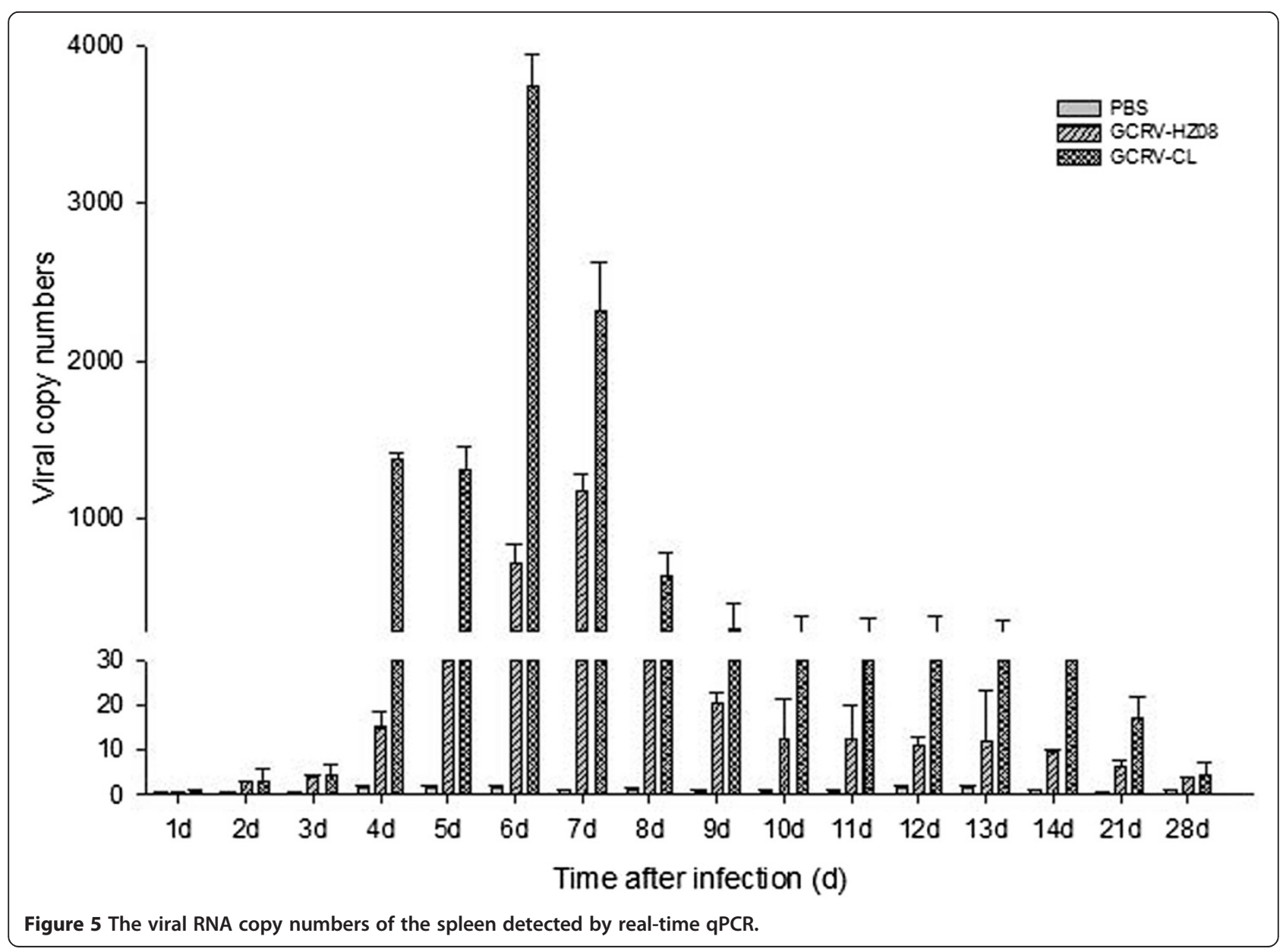


with FAM and on its 3' end with Eclipse. To estimate virus replication, virus-specific mRNA expression was measured using qRT-PCR and expressed as the number of RNA copies per mg of tissue. The results were analyzed using the Applied Biosystems 7500 software.

\section{Statistical analysis}

The experimental data were expressed as the mean \pm SD . The data were analyzed by a one-way ANOVA analysis and Student's t-tests using the SPSS 13.0 statistical software.

\section{Results}

Immunofluorescence analysis

GSB cells were infected with GCRV-HZ08 or GCRV-CL and the number of infected cells was determined using a fluorescence microscope (Figure 1). Both of the GCRVstains could propagated in the GSB cells.

\section{Fish pathogenicity experiments}

Grass carps infected with GCRV-CL by i.p. showed spots or plate hemorrhages in the tissues or organs and fish died, such as hemorrhages at the base of fins, intestines, and muscle (Figure $2 \mathrm{C} / \mathrm{F} / \mathrm{G} / \mathrm{H}$ ). However, grass carps infected with GCRV-HZO8 (Figure 2B/E) or the PBS group (Figure 2A/D) did not show clinical signs of this disease. GCRV-CL strain was more virulent than the GCRV-HZ08 strain.

\section{Detection of antibody}

The blood of three grass carps from each group were sampled, and the titers of the serum were determined using ELISA (Figure 3). The groups infected by GCRV-HZ08 and GCRV-CL showed elevated antibody levels, which suggested that GCRV-HZ08 and GCRV-CL had infected the grass carp and induced the elevated antibody levels.

\section{Tissue distribution of grass carp reovirus}

Three grass carps from each group were sampled from the first to fourteenth day and then on the twenty-first and twenty-eighth days. The viral RNA copy numbers of the samples in different tissues and at different times were detected by real-time qPCR.

\section{Liver}

To determine the viral RNA copy numbers in the liver, samples of liver were analyzed by real-time qPCR

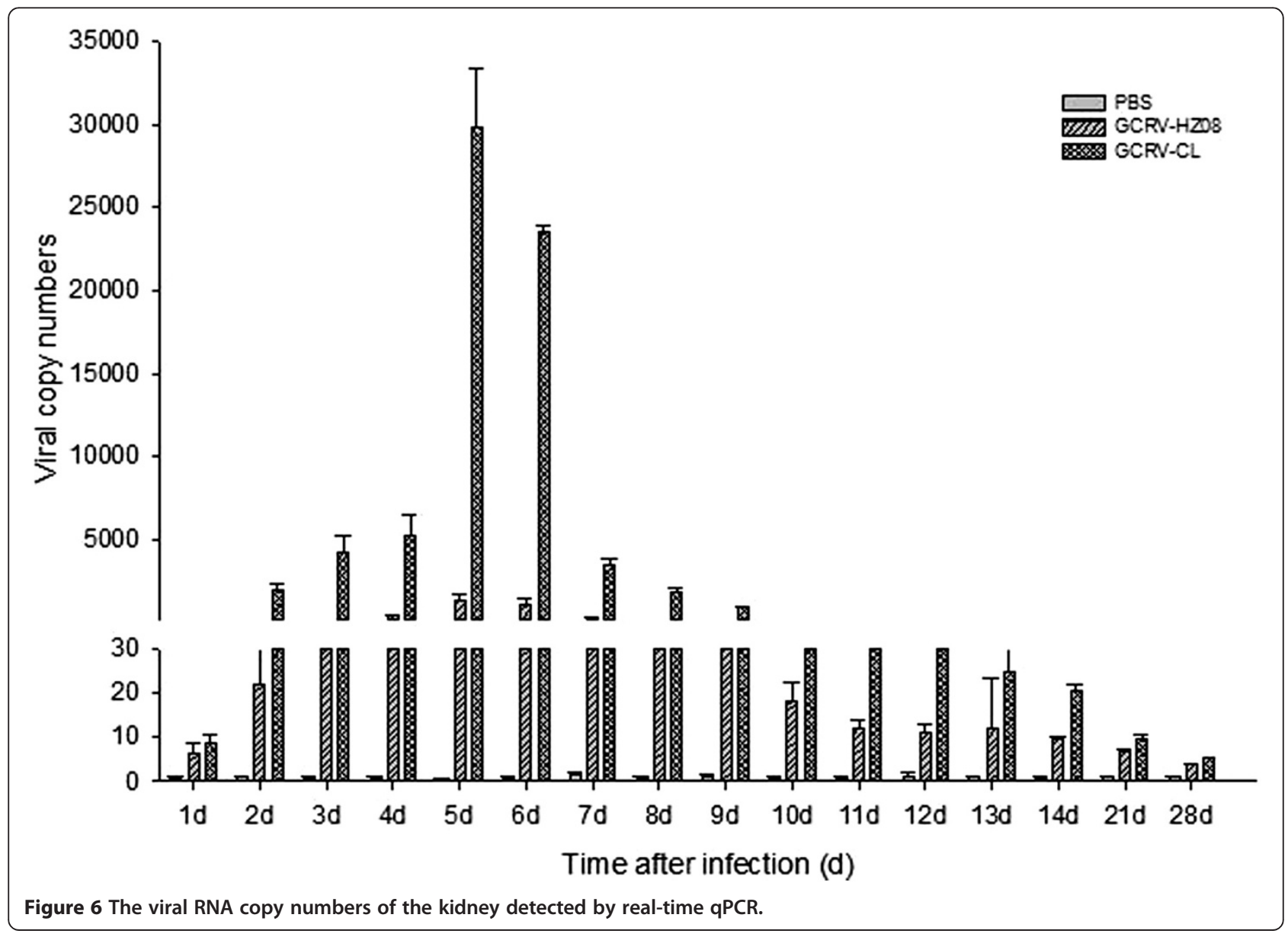


(Figure 4). The results showed that grass carps infected with GCRV-CL had the highest number of virus on the eighth day after infection, and the numbers on the seventh and ninth days were also high. No more than 30 viral copy numbers could be detected for GCRV-CL on the other days of infection or for GCRV-HZ08 on all days after infection.

\section{Spleen}

The viral RNA copy numbers of the spleen were detected using real-time qPCR (Figure 5). The results showed a higher number of GCRV-CL from the fourth to eighth days, whereas GCRV-HZ08 had a higher number of virus only on the sixth and seventh days, and the viral RNA copy numbers were less than that of GCRVCL. Moreover, the highest number of virus was for GCRV-CL on the sixth day.

\section{Kidney}

To determine the viral RNA copy numbers of the kidney, the samples were analyzed by real-time qPCR (Figure 6). The results showed that GCRV-CL had a higher viral number from the second to ninth day and the highest number on the fifth day. GCRV-HZ08 had a higher number of virus from the fourth to seventh day.

\section{Intestine}

The viral RNA copy numbers of the intestine were determined by real-time qPCR (Figure 7). The results showed that GCRV-CL had a higher number of virus from the fifth to tenth day and the highest number of virus on the seventh day. GCRV-HZ08 did not have a higher number of virus on any day.

\section{Muscle}

To determine the viral RNA copy numbers in the muscle, muscle samples were analyzed by real-time qPCR (Figure 8). The results showed that GCRV-CL had a higher number of virus from the sixth to tenth day and the highest number on the seventh day. GCRVHZ08 had a higher number of virus in the seventh day.

\section{Discussion}

GCRV is a multilayered, spherically structured particle that contains a genome of 11 segments of dsRNA (named S1-S11) that encode 7 structural (VP1-VP7)

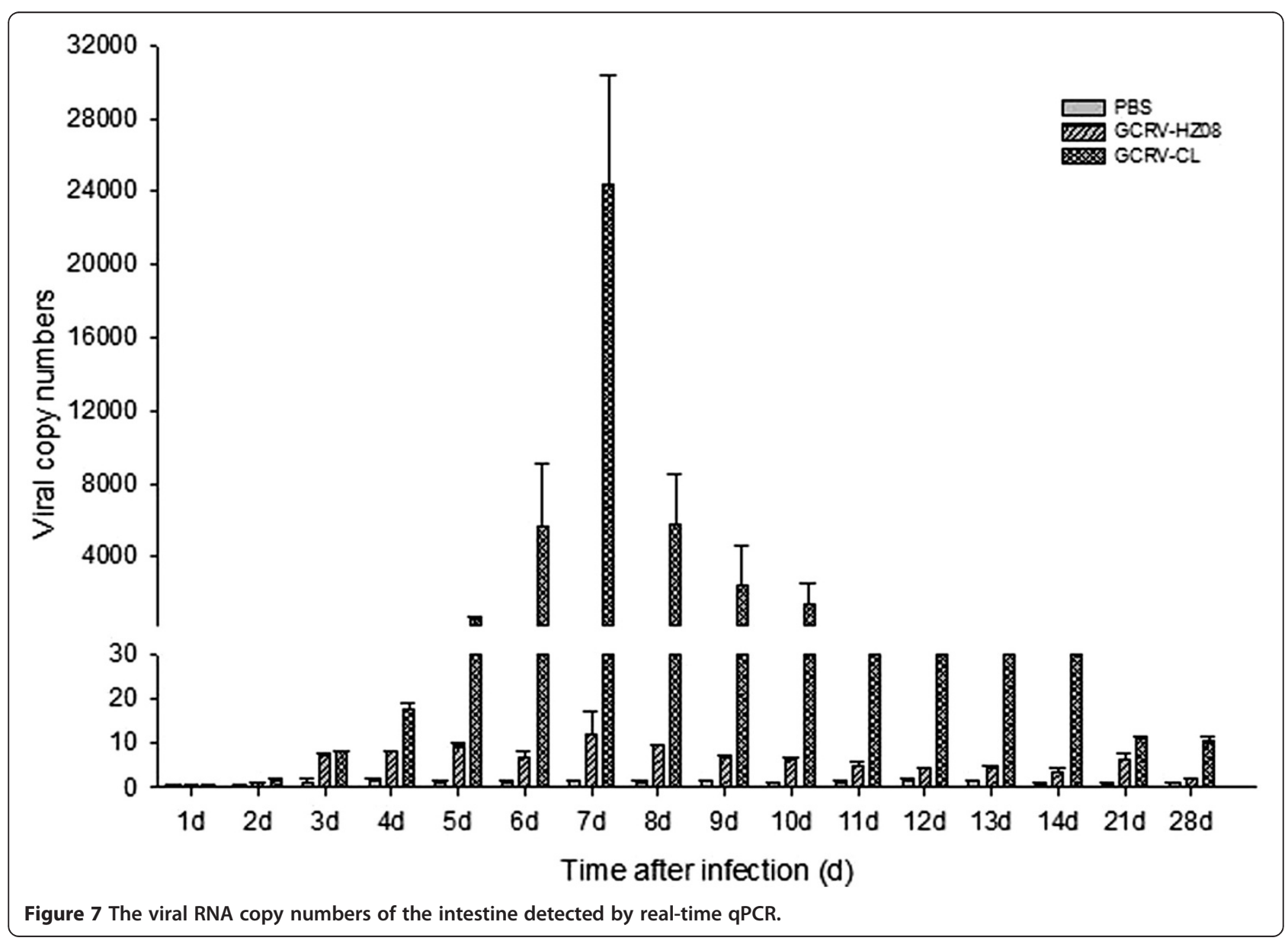




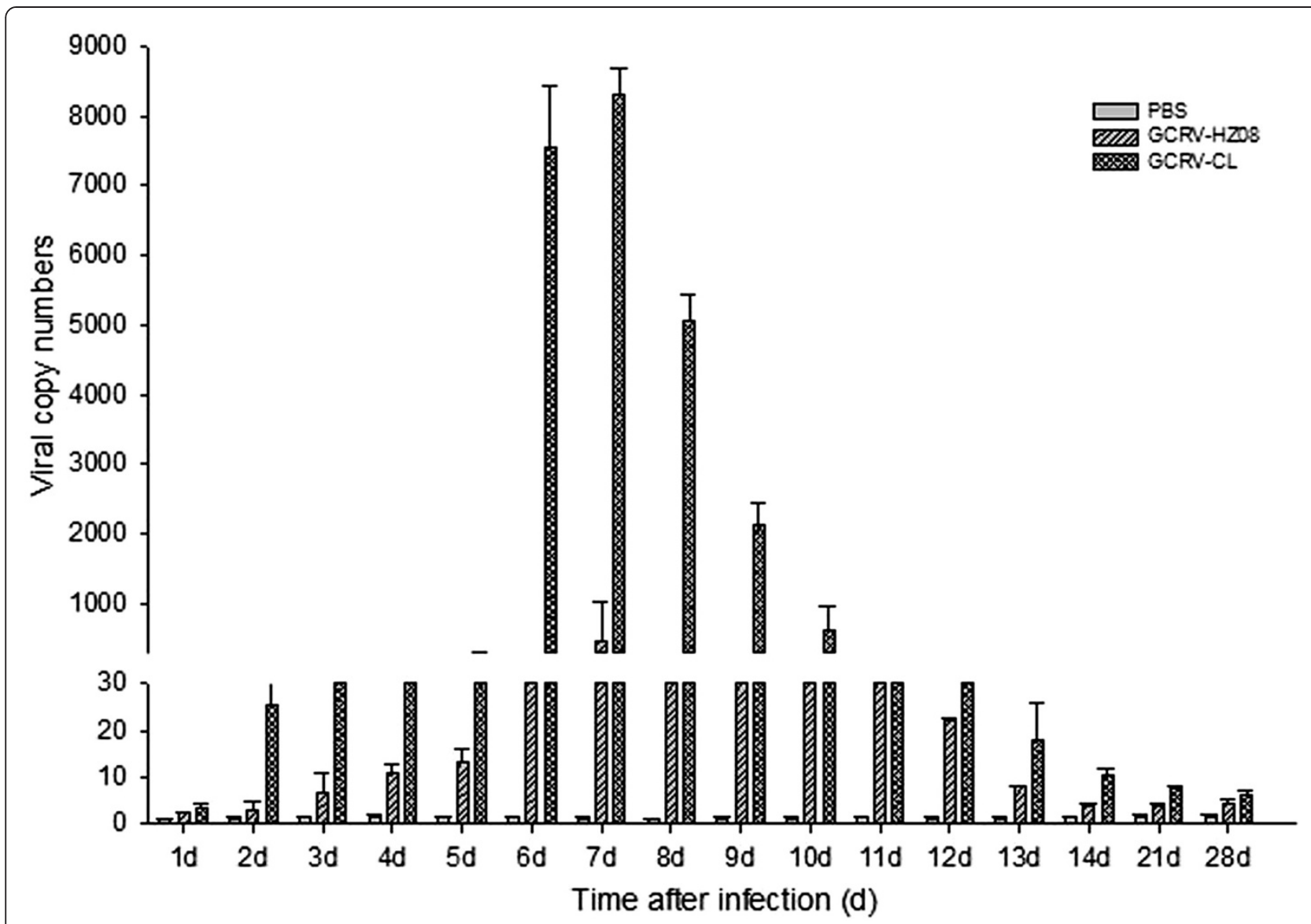

Figure 8 The viral RNA copy numbers of the muscle detected by real-time qPCR.

and 5 nonstructural proteins [10]. But it is still limited to know the diseases [3].

Most attenuated virus strains replicate quickly and express large amounts of antigen protein, thereby inducing strong adaptive immune responses that result in rapid virus clearance. But pathogenic virus strains replicate at a lower rate than attenuated strains and they could reach their target tissues $[11,12]$. In this study, GCRV-CL was a pathogenic strain, and GCRV-HZ08 was an attenuated virus strain. Hence, GCRV-HZ08 may be cleared from the body more easily, which could explain why GCRVHZ08 was undetectable or only detected for a few days by qRT-PCR.

The clinical signs of this infection are hemorrhages of organs, showing spots or plate forms, in combination with some or all of the following signs: exophthalmia, body darkening, hemorrhage of the mouth cavity, hemorrhagic or pale gills, and hemorrhage at the base of fins and gill covers. Internal hemorrhages may occur throughout the musculature, liver, spleen, kidney, and intestines. However, these are only some of the signs; and sick fingerlings show one or all of them [13]. The results of this study were consistent with the above- mentioned study. Grass carps infected with GCRV-CL by i.p. showed spots or plate hemorrhages in the tissues or organs, such as hemorrhage at the base of fins, intestines, and muscle, but grass carps infected with GCRVHZ08 did not show any of the clinical signs (Figure 2). GCRV-HZ08 could have been attenuated after cell passaging; therefore, the GCRV-CL strain was more virulent than the GCRV-HZ08 strain. Western blotting showed elevated antibody levels and suggested that GCRV-CL and GCRV-HZ08 had infected the grass carps and induced the high antibody titers (Figure 3 ).

In this study, the viral RNA copy numbers were detected by qRT-PCR, and threshold cycles $(\mathrm{Ct})$ for each sample that were lower than $35(\mathrm{Ct}<35$; copy $>20)$ were treated as positive. Grass carp infected with GCRV-CL showed higher numbers of viral RNA copies during the sixth to ninth day in nearly all tissues, indicated that the seventh day was the peak incidence. These four days had at least more than 1000 copies. The kidney had the earliest highest numbers of viral RNA copies, which were as high as 24,000 copies on the fifth day. The kidney may be a region of virus replication, serving as a factory to produce a large number of GCRV that are then spread 
to other parts of the body. Ten days after infection, the viral RNA copy numbers gradually decreased, and until the fourteenth day, nearly no viral RNA copies could be detected, possibly because GCRV-CL was gradually cleared from the body. The virus may have been eliminated in the fourteenth day.

In conclusion, this study defined the virus distribution of different tissues of grass carp inoculated by i.p. and supplies clues for the pathogenesis of GCRV. However, further studies should be performed to better understand the tropism and transmission of GCRV.

\section{Conclusions}

Two different strain of GCRV:GCRV-CL and GCRVHZ08 were infected grass carp. Grass carps infected byGCRV-CL showed spots or plate hemorrhages in the tissues and was more virulent than the GCRV-HZ08 strain. The grass carps infected by GCRV-CL had higher viral RNA copy numbers than infected by GCRV-HZ08 in different tissues and organs, particularly in the liver, spleen, kidney, intestine, and muscle, which had a higher number of viral RNA copies during the sixth to ninth days. But ten days after infected, there were not high viral RNA copy numbers. This study defined the virus distribution of different tissues of grass carp inoculated by i.p. and supplies clues for the pathogenesis of GCRV.

\section{Competing interests}

The authors declare that they have no competing interests.

\section{Authors' contributions}

$H-R L, Y-G L$ and QW performed lab experiments. W-WZ and Y-YW analyzed the data. S-QW conceived and designed the experiments. $\mathrm{H}-\mathrm{RL}$ wrote the manuscript. All authors have read and approved the final manuscript.

\section{Acknowledgments}

This project was supported by the National Key Technology R\&D Program (Grant No. 2012BAD25B02), the Special Scientific Research Funds for Central Non-profit Institutes, Chinese Academy of Fishery Sciences (Grant No. 2014A06XK09), the National Natural Science Fund (No.31202026) and the earmarked fund for the China Agriculture Research System (Grant No. CARS-46).

\section{Author details}

${ }^{1}$ Pearl River Fisheries Research Institute, Chinese Academy of Fishery Sciences, Key Laboratory of Fishery Drug Development,Ministry of Agriculture, Key Laboratory of Aquatic Animal Immune Technology, Guangzhou 510380, China. ${ }^{2}$ College of Fisheries and Life Science, Shanghai Ocean University, Shanghai 201306, China.

Received: 5 June 2014 Accepted: 15 September 2014 Published: 7 October 2014

\section{References}

1. Chen C, Sun X, Liao L, Luo S, Li Z, Zhang X, Wang Y, Guo Q, Fang Q, Dai H: Antigenic analysis of grass carp reovirus using single-chain variable fragment antibody against IgM from Ctenopharyngodon idella. Sci China Life Sci 2013, 56:59-65.

2. Wang Q, Zeng W, Liu C, Zhang C, Wang Y, Shi C, Wu S: Complete genome sequence of a reovirus isolated from grass carp, indicating different genotypes of GCRV in China. J Virol 2012, 86:12466.

3. Tao Qiu R-HL, Zhang J, Zhu Z-Y: Complete nucleotide sequence of the S10 genome segment of grass carp reovirus (GCRV). Dis Aquat Organ 2001, 44:69-74.
4. Shao L, Sun X, Fang Q: Antibodies against outer-capsid proteins of grass carp reovirus expressed in $\mathrm{E}$. coli are capable of neutralizing viral infectivity. Virol J 2011, 8:347.

5. Fan C, Zhang L-I, Lei C-f, Fang Q: Expression and identification of inclusion forming-related domain of NS80 nonstructural protein of grass carp reovirus. Virol Sin 2009, 24:194-201.

6. Ma J, Wang W, Zeng L, Fan Y, Xu J, Zhou Y: Inhibition of the replication of grass carp reovirus in CIK cells with plasmid-transcribed shRNAs. J Virol Methods 2011, 175:182-187.

7. Cheng L, Fang Q, Shah S, Atanasov IC, Zhou ZH: Subnanometer-resolution structures of the grass carp reovirus core and virion. J Mol Biol 2008, 382:213-222.

8. Yan L, Guo H, Sun X, Shao L, Fang Q: Characterization of grass carp reovirus minor core protein VP4. Virol J 2012, 9:89.

9. Cai L, Sun X, Shao L, Fang Q: Functional investigation of grass carp reovirus nonstructural protein NS80. Virol J 2011, 8:168.

10. Zhang L, Luo Q, Fang Q, Wang Y: An improved RT-PCR assay for rapid and sensitive detection of grass carp reovirus. J Virol Methods 2010, 169:28-33.

11. Faber M, Li J, Kean RB, Hooper DC, Alugupalli KR, Dietzschold B: Effective preexposure and postexposure prophylaxis of rabies with a highly attenuated recombinant rabies virus. Proc Natl Acad Sci U S A 2009, 106:11300-11305.

12. Jinyang Zhang X, Zan J, Wu Y, Chengjin Y, Ruan X, Zhoua J: Cellular chaperonin CCT contributes to rabies virus replication during infection. J Virol 2013, 87(13):7608-7621.

13. Jiang $Y$ : Hemorrhagic disease of grass carp status of outbreaks, diagnosis, surveillance, and research. Bamidgeh 2009, 61:188-197.

doi:10.1186/1743-422X-11-178

Cite this article as: Liang et al:: Pathogenicity and tissue distribution of grass carp reovirus after intraperitoneal administration. Virology Journal 2014 11:178.

\section{Submit your next manuscript to BioMed Central and take full advantage of:}

- Convenient online submission

- Thorough peer review

- No space constraints or color figure charges

- Immediate publication on acceptance

- Inclusion in PubMed, CAS, Scopus and Google Scholar

- Research which is freely available for redistribution 